caring for an ill spouse is still a big burden in current rural China though family-based assistance acts as functional solidarity for older people, especially for widowers.

\section{WORK-FAMILY CONFLICT AT MIDLIFE: FAMILY ELDERCARE AND MENTAL HEALTH OF MIDDLE- AGED WORKERS IN CHINA}

Z. Lin, University of Maryland, College Park

As the population ages worldwide, work-family conflict extends to midlife. Although it is widely known that caregivers for elderly parents are less likely to be in the labor market than non-caregivers, well-being of caregivers who stay on their jobs has not been fully explored. By using two waves of nationally representative longitudinal sample of middle-aged adults (aged 40 59) in China (2011 and 2013, $\mathrm{N}=10,153$ ), this paper examines 1) the prevalence of informal eldercare among middle-aged workers in a context where labor force participation keeps at a high level for both men and women while eldercare responsibilities are mainly taken by adult children; 2) health implications of eldercare for employed adult children. I firstly found that although employed daughters were still highly involved in eldercare $(29.7 \%$ in 2011 and $36.5 \%$ in 2013) and more likely to leave the labor market between two waves than their male counterparts, surprisingly, employed sons in China reported a similar level of eldercare involvement (29.9\% in 2011 and $36.7 \%$ in 2013). Secondly, results from multivariate analyses showed significant gender and rural-urban differences in mental health outcomes of parent eldercare among middle-aged workers. Employed daughters and sons who kept taking the eldercare role for both waves reported a higher level of depressive symptoms than those who were not involved in eldercare at any wave in rural China, whereas the converse was true for employed daughters in urban areas. Findings have important policy implications for supporting middle-aged workers to balance their employment and informal eldercare.

\section{SESSION 2245 (POSTER)}

\section{DEPRESSION AND PSYCHOSOCIAL CHARACTERISTICS III}

\section{A COMPARATIVE STUDY OF THE ROLE OF SOCIAL NETWORKS ON DEPRESSIVE SYMPTOMS}

N. Park ${ }^{1}$, Y. Jang ${ }^{2}$, S. Chung ${ }^{3}$, D. Chiriboga ${ }^{1}, 1$. University of South Florida, 2. The University of Texas at Austin, 3. Ewha Womans University

Based on the broken convoy variant of social convoy model (Kahn \& Antonucci, 1980; Park et al., 2015), this study examined the role of family and friend networks and other personal and cultural resources on depressive symptoms in three groups of Koreans/Korean Americans. Data were drawn from the surveys with older Koreans $(n=403)$ and Korean Americans in the New York City metropolitan area (high Korean density area; $\mathrm{n}=420$ ) and west central Florida (low Korean density area; $n=672$ ). It was hypothesized that different social contexts such as ethnic population density would predict depressive symptoms for the Korean and Korean American samples. Multiple regression models estimated the effects of socio-demographic characteristics (age > 75, female, living alone, perceived financial status, and fair/poor rating of health), social network (family network and friend network), and immigration-related characteristics for the two Korean American samples (the length of stay in the U.S. and acculturation). For older Koreans, neither family network nor friend network were associated with depressive symptoms but living alone and low perceived financial status were. For both Korean American samples, smaller family network, fair/poor rating of health, and lower levels of acculturation were associated with greater depressive symptoms. Additionally, lower perceived financial status for the New York sample and living alone for the Florida sample were linked with depressive symptoms. The results suggest that differential roles of social network, personal and cultural resources should be considered when working with older ethnic population in different social and environmental contexts.

\section{A MODERATED MEDIATION MODEL OF SELF- EFFICACY, CATASTROPHIZING AND DEPRESSIVE SYMPTOMS IN CHRONIC PAIN PATIENTS}

M. Leung, S. Cheng, The Education University of Hong Kong

Self-efficacy has been consistently found to be a protective factor against psychological distress and disorders in the literature. However, little research is done on the moderating effect of self-efficacy on depressive symptoms in the context of chronic pain. This cross-sectional study aimed to examine if pain self-efficacy attenuated the direct relationship between pain intensity and depressive symptoms, as well as their indirect relationship through reducing the extent of catastrophizing when feeling pain (moderated mediation). 664 community-dwelling Chinese older adults aged 60-95 years who reported chronic pain for at least three months were recruited from social centers. They completed a battery of questionnaires on chronic pain, pain self-efficacy, catastrophizing, and depressive symptoms in individual face-to-face interviews. Controlling for age, gender, education, self-rated health, number of chronic diseases, pain disability, and pain self-efficacy, pain catastrophizing was found to partially mediate the connection between pain intensity and depressive symptoms. Furthermore, the relationship between pain intensity and depressive symptoms was moderated by pain self-efficacy. Self-efficacy was also found to moderate the relationship between pain intensity and catastrophizing and the moderated mediation effect was confirmed using bootstrap analysis. The results suggested that pain intensity's direct effect on depressive symptoms and its indirect effect on depressive symptoms via catastrophizing both disappeared when self-efficacy was high. Our findings suggest that pain self-efficacy is a significant protective factor that contributes to psychological resilience in chronic pain patients by attenuating the relationship of pain intensity to both catastrophizing and depressive symptoms.

\section{ASSESSING THE ROLE OF SELECTION BIAS IN THE PROTECTIVE RELATIONSHIP BETWEEN CAREGIVING AND MORTALITY}

M. Smith, T. Heeren, L. Ranker, L. Fredman, Boston University School of Public Health

Selection bias is a concern in studies of caregiving outcomes. In contrast to predictions based on stress theory, 
most population-based studies have found lower rates of mortality in caregivers than non-caregivers. We examined the potential role of selection bias due to 1) study design and 2) selective participation in Caregiver-SOF, an ancillary study to the Study of Osteoporotic Fractures (SOF). Caregiver-SOF includes 1069 SOF participants (375 caregivers, each matched to 1-2 non-caregivers) identified in two phases: screening all SOF participants for caregiver status at SOF Visit 6 (1997-1999, n=4036 women, 23\% caregivers) and rescreening all caregivers and a subset of non-caregivers matched on sociodemographic factors 1-2 years later. Mean age at initial screening was 79 years. Older women and women with poorer physical or cognitive functioning were less likely to participate. Caregivers had better functioning than non-caregivers at each screening. We calculated adjusted hazards ratios (aHR) to assess associations between caregiving and 10-year mortality in all 4036 initially screened women, women invited to participate (all caregivers and selected matched non-caregivers, $n=1449)$, and the Caregiver-SOF sample $(74 \%$ of those invited). Adjusting for functioning and matching variables, the association between caregiving and mortality in invited women $(48 \%$ died; aHR $=0.79 ; 95 \% \mathrm{CI}: 0.65-0.97)$ was similar to that in initially screened women $(37 \%$ died; $\mathrm{aHR}=0.84 ; 95 \% \mathrm{CI}: 0.73-0.96)$, indicating minimal bias due to study design, and to that in Caregiver-SOF $(48 \%$ died; $\mathrm{aHR}=0.80 ; 95 \% \mathrm{CI}: 0.63-1.03)$, indicating minimal participation bias. These results lend validity to findings that caregivers have lower risk of mortality.

\section{ASSOCIATION BETWEEN PERCEIVED SOCIAL SUPPORT FROM DIFFERENT SOURCES AND SELF- MASTERY AMONG U.S. CHINESE OLDER ADULTS \\ S. Zhang, X. Dong, Rush University Medical Center}

Background: Researchers have examined the relationship between perceived social support (SS) and personal self-mastery (SM), but there is limited knowledge about this association among immigrant populations who may have less available external social support. This study examines the association between SS and SM among U.S. Chinese older adults. Methods: Data were derived from 3,157 Chinese older adults age 60 and over from the PINE study. SS was measured with 12 items from NSHAP regarding positive/negative SS from spouse, family, and friends. Overall SS is the sum of positive and negative support. SM was assessed by the 7-item Pearlin Mastery Test. Linear regression was conducted adjusting for confounders. Results: The mean SM of study participants was $34.6 \pm 7.7$ (range: $7-49$ ). Higher overall SS from spouse $(b=0.94, S E=0.1)$, family $(b=0.93, S E=0.1)$, and friends $(b=0.94, S E=0.1)$ were associated with higher SM. Further, higher positive SS from spouse $(b=1.1, S E=0.13)$, family $(b=0.74, S E=0.1)$, and friends $(b=0.87, S E=0.1)$ were also associated with higher SM. On the other hand, higher negative SS from spouse $(b=-0.97, S E=0.18)$, family $(b=-$ 1.89, $\mathrm{SE}=0.24)$, and friends $(\mathrm{b}=-1.43, \mathrm{SE}=0.42)$ were associated with lower SM. Conclusions: Our study shows both positive SS and negative SS are related to SM among U.S. Chinese older adults. Further longitudinal studies are needed to identify modifiable factors to improve SS and SM among immigrant populations.

\section{DEPRESSIVE SYMPTOMS AND CHRONIC DISEASE: IS THERE AN ASSOCIATION FOR OLDER AFRICAN AMERICANS?}

S. Cho, T. Hamler, Case Western Reserve University

The purpose of this study was to identify the association between physical health problems and depressive symptoms among older African Americans (age > 65) using the 2014 Health and Retirement Study $(\mathrm{N}=1,206)$. Membership in a racial/ethnic minority group may restrict access to mental health services. Many existing studies have reported the higher prevalence of depressive symptoms among older minority populations. Racial/ethnic minority groups, particularly African Americans experience a disproportionate burden of chronic conditions, as well as disparities accessing health care and preventive services due to a variety of socioeconomic, behavioral, and other factors. For statistical analysis, depressive symptoms were measured with eight-item CES-D Scale $(\mathrm{M}=1.546, \mathrm{SD}=1.952)$, and chronic health conditions were measured by asking whether respondents had ever diagnosed with the following seven diseases: high blood pressure, diabetes, cancer, lung disease, heart disease, stroke, and arthritis $(\mathrm{M}=2.736, \mathrm{SD}=1.285)$. Sociodemographic covariates included self-reported health, age, gender, income (logged), marital status, and educational attainment. An OLS regression showed that older African Americans having one additional chronic health problem was associated with .133 higher depressive symptoms after controlling for the covariates $(\mathrm{p}=.002)$. The regression model could be an appropriate prediction on depressive symptoms, Adjusted $\mathrm{R}$-square $=.161, \mathrm{~F}(7,1,198)=34.127, \mathrm{p}<.001$. Depression and chronic illness together are drivers that increase healthcare costs and have developed into a critical problem for an already vulnerable older African American population, requiring a critical attention from both practice and policy.

\section{DOES COPING MATTER? COPING AND CHRONIC PHYSIOLOGICAL DISTRESS AMONG AGING U.S. BLACK AND WHITE MEN}

J. Allen ${ }^{1}$, D. Watkins ${ }^{2}$, L. Chatters ${ }^{3}$, V. Johnson-Lawrence ${ }^{4}$, 1. University of Michigan, 2. University of Michigan School of Social Work, 3. University of Michigan Schools of Public Health and Social Work, 4. Michigan State University

Division of Public Health

Chronic physiological distress accelerates biological aging and is linked to chronic disease and premature mortality. Physiological distress biomarkers, such as cortisol, represent a key proximal outcome of stress and coping processes through which social inequities are posited to generate health disparities. This study explored how variations in coping may contribute to documented racial disparities in chronic physiological distress between midlife and older Black and White male participants in the National Survey of Midlife Development in the United States (MIDUS) II and the National Study of Daily Experiences (NSDE) II. Black and White men generally reported using similar coping strategies, though Black men relied more on positive reinterpretation, denial, drug use, and physical inactivity than White men. Of the 12 coping strategies examined, ten were unrelated to men's chronic physiological distress levels. Religious and spiritual coping appeared protective, but only for White men. Black men who reported drug use (i.e., illegal drugs, 